\section{Social media}

\section{Clip-on caution}

Sir, as a young dentist, I am no doubt guilty of spending too much time browsing social media. Over the past year, I have seen a huge increase in targeted advertisements covering dental services and products. While some appear to be relatively harmless, I was shocked by a recent advertisement for 'Clip-on veneers'. Although a recent letter to the $B D J$ raised similar concerns, ${ }^{1}$ these dangerous products must be made known to dental professionals and thus this subject warrants further attention.

Clip-on veneers are offered by multiple companies based in the UK and beyond, and are made using an at-home impression kit. After taking their upper and lower impressions using the provided trays (no bite registration required), consumers send them back to the manufacturers along with their selected shade of BL1 or BL3 so that their 'veneers' can be manufactured.

There are a number of concerning elements of these products which are immediately obvious to a dental professional, such as the potential to cause occlusal, periodontal and carious issues. Not only may these products cause detriment to a patient's oral health, they can also disguise active disease and prevent patients from seeking dental treatment when necessary. Despite these issues, our patients may see clip-on veneers as a quick and cheap solution to get straight white teeth, not knowing the potential damage they can cause.

Rather than outlining these painfully blatant issues, I would instead recommend that readers do a quick Google search of 'clip-on veneers Instagram' to see these products for themselves. In the featured before and after photographs, there are multiple cases which demonstrate extensive untreated carious lesions and severe periodontal disease. I doubt that the provision of clip-on veneers will do much to help these conditions.

The GDC functions to regulate dentistry, and should thus be actively seeking out and regulating dangerous practices and products to ensure that our patients are kept safe. Indeed, in February 2020, the GDC stated that they were 'continuing to gather evidence about the potential risk of harm' of 'forms of dental care offered remotely' in order to regulate direct-to-consumer products. ${ }^{2}$ I wonder what the cause for our regulator's delay in cracking down on these products is, and whether they should be doing more to protect our patients?

\section{R. Gallagher, Edinburgh, UK}

\section{References}

1. Eaton J. Clip-on questions. Br Dent J 2020; 230: 325

2. General Dental Council. Providing dental care remotely. 2020. Available at: https://www.gdc-uk.org/newsblogs/news/detail/2020/02/24/providing-dental-careremotely (accessed August 2021).

https://doi.org/10.1038/s41415-021-3486-x

\section{Pharmaceuticals}

\section{Betamethasone vigilance}

Sir, the use of betamethasone 500 microgram soluble tablets as a mouthwash is referenced in national guidance sources as a treatment for oral ulceration, including aphthous ulcers. ${ }^{1,2}$ However, the topical use of this medication is unlicensed. The Summary of Product Characteristics for betamethasone 500 microgram soluble tablets advises that: 'Betnesol Tablets/ Betamethasone Tablets are best taken dissolved in water, but they can be swallowed whole without difficulty' ${ }^{3}$ The advice in the Patient Information Leaflet (PIL) which is provided with the product states: 'Your tablets can be swallowed whole, but they are best taken as a drink after allowing them to dissolve in a glass of water. The PIL also advises to 'always take this medicine exactly as your doctor or pharmacist has told you.' ${ }^{4}$

The National Patient Safety team at NHS England and NHS Improvement has received a number of incident reports related to betamethasone soluble tablets prescribed as a mouthwash but mistakenly taken orally. One report described hospital admission for adrenal crisis, a potentially life-threatening condition, particularly if not recognised early. ${ }^{5}$ To reduce the risk of inappropriate use, dental and medical practitioners prescribing betamethasone 500 microgram soluble tablets for use as a mouthwash need to include detailed instructions on the prescription and also provide information to the patient. The authors would recommend that prescriptions are endorsed, with full dose instructions - ie ' 500 micrograms four times a day, to be dissolved in 10-20 ml water and rinsed around the mouth' but include 'for use as a mouthwash' and 'do not swallow', 1,6 Directions on the prescription will always be replicated on the dispensing label.

The British and Irish Society for Oral Medicine (BISOM) has a detailed and comprehensive PIL on the use of betamethasone mouthwash and this can be accessed and downloaded from the society website. ${ }^{6}$ The authors advise that the patient receives the BISOM PIL - in addition to the manufacturers' PIL supplied with the medicine.

A. Field, C. Randall, Liverpool, UK

\section{References}

1. BNF. Treatment of aphthous ulcers. Available at: https:// bnf.nice.org.uk/treatment-summary/oral-ulcerationand-inflammation.html (accessed September 2021).

2. NICE Clinical Knowledge Summaries. Aphthous ulcer. Available at: https://cks.nice.org.uk/topics/aphthousulcer/ (accessed September 2021).

3. Summary of Product Characteristics. RPH Pharmaceuticals AB. Betamethasone 500 microgram Soluble Tablets. Available at: https://www.medicines. org.uk/emc/product/9098/smpc (accessed September 2021).

4. Patient Information Leaflet. RPH Pharmaceuticals AB. Betamethasone 500 microgram Soluble Tablets. Available at: https://www.medicines.org.uk/emc/ product/9098/pil (accessed September 2021)

5. Specialist Pharmacy Service. Understanding safety risks with betamethasone soluble tablets used as mouthwash. 2021. Available at: https://www.sps. nhs.uk/articles/understanding-safety-risks-withbetamethasone-soluble-tablets-used-as-mouthwash/ (accessed September 2021).

6. The British and Irish Society for Oral Medicine. Patient information leaflet - Betamethasone 500 microgram soluble tablets used as a mouthwash. Available at: https://bisom.org.uk/wp-content/uploads/2020/02/ Betamethasone-soluble-tablets-used-as-mouthwashPIL-October-2019.pdf (accessed September 2021).

https://doi.org/10.1038/s41415-021-3487-9

\section{Evidence-based dentistry}

\section{Halting 'research waste'}

Sir, the main goal of evidence-based dentistry (EBD) is to integrate the most up-to-date and reliable scientific evidence with dentists' clinical expertise, to drive up standards of care. ${ }^{1}$ Recently, numerous studies have published questionable findings, ${ }^{2,3,4}$ including an overwhelming number of COVID-19 clinical trials without adequate methodological rigour and design. This is contrary to the principles of EBD. ${ }^{1,5}$

Such occurrences raise the notion of a well-known phenomenon in medical sciences: 'research waste. ${ }^{4}$ Dentistry is no exception, where it has been recognised by many. ${ }^{5}$ Despite previous attempts to enhance the quality of dental evidence, several questions with significant 